\title{
Los derechos procesales en grupos vulnerables desde la normativa nacional e internacional $^{1}$
}

Procedural rights in vulnerable groups from national and international regulations

\author{
Yahaira Berenice, Martínez-Pérez ${ }^{2}$ \\ (iD) https://orcid.org/0000-0002-4047-7298 \\ Brenda Judith, Sauceda-Villeda ${ }^{3}$ \\ https://orcid.org/0000-0003-3614-7875 \\ María Salomé, Moreno-Rodríguez ${ }^{4}$ \\ (iD) https://orcid.org/0000-0001-8106-1910 \\ Universidad Autónoma de Nuevo León, México \\ https://doi.org/10.29105/pgc6.12-12
}

\section{RESUMEN}

El presente trabajo es producto de una revisión documental que tuvo por objetivo analizar los derechos procesales en los grupos vulnerables desde el marco nacional e internacional. El estudio enfoca un análisis de tipo reflexivo y analítico jurídico. Se aplicó un método heurístico de categoría taxonómico en metodología cualitativa. Se ha desarrollado en dos fases: la primera refiere el marco teórico-jurídico y la segunda busca señalar los derechos procesales de los grupos vulnerables en los procesos judiciales. En el estudio reflexivo, se observó la existencia de la vulnerabilidad primaria y secundaria. Se concluye la necesidad de generar un protocolo para la atención de derechos procesales en los grupos vulnerables en protección de los derechos humanos.

Palabras clave: Derechos humanos, grupos vulnerables, vulnerabilidad

\begin{abstract}
This work is the product of a documentary review that aimed to analyze procedural rights in vulnerable groups from the national and international framework. The study focuses on a reflective and analytical legal analysis. A taxonomic category heuristic method was applied in qualitative methodology. It has been developed in two phases: the first refers to the theoretical-legal framework and the second seeks to point out the procedural rights of vulnerable groups in judicial processes. In the reflective study, the existence of primary and secondary vulnerability was observed. The need to generate a protocol for the attention of procedural rights in vulnerable groups in protection of human rights is concluded.
\end{abstract}

Keywords: Human rights, vulnerable groups, vulnerability.

Recibido: 04 de Diciembre 2019 - Aceptado: 07 de Abril 2020 - Corregido: 10 de Mayo 2020

Cómo referenciar este artículo:

Martínez-Pérez, Y. B., Sauceda-Villeda, B. J. \& Moreno-Rodríguez, M. S. (2020). Los derechos procesales en grupos vulnerables desde la normativa nacional e internacional. Politica Globalidad y Ciudadanía, 225-245. Recuperado de http://revpoliticas.uanl.mx/index.php/RPGyC/article/view/156

\footnotetext{
1 Este artículo es derivado del proyecto de investigación "La ausencia de unicidad de criterios normativos y jurisprudenciales reguladores del debido proceso del adulto mayor en el juicio administrativo a nivel federal", cuenta con financiamiento de CONACYT y la Universidad Autónoma de Nuevo León.

2 Doctora en Métodos Alternos y Solución de Conflictos por la Universidad Autónoma de Nuevo León, Profesor titular de la Facultad de Derecho y Criminología de la Universidad Autónoma de Nuevo León. Email: yahaira.martinezpr@uanl.edu.mx

3 Doctora en Métodos Alternos y Solución de Conflictos por Universidad Autónoma de Nuevo León, Profesor titular de la Facultad de Derecho y Criminología de la Universidad Autónoma de Nuevo León.Email:judith_sauceda@hotmail.com

4 Doctorante en Derecho Procesal de la Universidad Autónoma de Nuevo León, Profesor catedra de la Facultad de Derecho y Criminología de la Universidad Autónoma de Nuevo León. Email: salome.morenordz@uanl.edu.mx
} 


\section{1.- INTRODUCCIÓN}

En el presente artículo se presenta un análisis de los derechos procesales en grupos vulnerables en la normativa nacional e internacional. En virtud del cual se realiza una metodología cualitativa a través de la heurística. En este sentido, se observa la necesidad de describir los derechos procesales y los grupos vulnerables en el esquema de garantía constitucional y universal. Por consiguiente, los primeros antecedentes del Derecho se remiten a la época de los romanos, se determina el jus como el conjunto de normas establecida por el Estado imperativas para los ciudadanos.

El derecho procesal es la ciencia del derecho que integra diversos estudios en el sistema jurídico, en los tribunales de justicia y en las normas de procedimiento para efectos de garantizar los derechos de las personas ante los órganos jurisdiccionales (Colombo Campbell, 2002). En este orden de ideas, el derecho procesal se entiende como la disciplina que estudia de manera sistemática la organización jurisdiccional del Estado y la impartición de justicia.

Así mismo, se plantea una preocupación en los derechos procesales de aquellos grupos que por alguna circunstancia son etiquetados por la comunidad como débiles, poniéndolos en un nivel de desventaja en un estado de inferioridad y exclusión social, es decir, vulnerables a la segregación lato sensu (Peláez Grisales, 2015).

Ahora bien, en miras de fortalecer la dignidad de las personas, en el año 2011 la Constitución Política de los Estados Unidos Mexicanos en adelante CPENM, estableció una reforma al artículo $1^{\circ}$. en materia de los Derechos Humanos (H. Congreso de la Unión), lo cual ha generado altas expectativas en los procesos judiciales en garantía a los derechos humanos. En el marco internacional la Declaración Universal de los Derechos Humanos, la Convención Americana sobre Derechos Humanos en Materia de Derechos Económicos, Sociales y Culturales "Protocolo de San Salvador" y el Pacto Internacional de Derechos Civiles y Políticos, son reconocidos como los principales instrumentos de carácter universal donde se sustentan los derechos esenciales de las personas.

Si bien las normas jurídicas aplicables de contexto nacional y universal establecen garantías sobre los derechos de los grupos vulnerables, cabe advertir que datos estadísticos expedidos por la Comisión Nacional de los Derechos Humanos (2018), determinan la existencia de continuas violaciones a los derechos humanos afectando en su mayoría a las poblaciones vulnerables en cuanto se carece de un mecanismo incorporado en la norma jurídica de atención a los derechos procesales de los grupos vulnerables. En este sentido, cabe reflexionar ¿Cuáles son los derechos procesales en los grupos vulnerables desde la normativa nacional e Internacional? 
Por lo anteriormente descrito, hemos considerado la importancia de identificar los derechos procesales de los vulnerables, para efectos de garantizar el respeto a los derechos humanos. Ahora bien, es necesario distinguir quienes son los grupos vulnerables, dado el contexto de discriminación lato sensu que acoge a esta población en los procedimientos judiciales. También es importante señalar el marco jurídico nacional e internacional, en este corpus iuris se sustentan los derechos fundamentales que garantizan el principio de igualdad y con ello el acceso a la justicia.

En este artículo, se pretende analizar los derechos procesales en los grupos vulnerables desde el contexto legal, se busca conocer la clasificación jurídica y doctrinal que enmarca a la población vulnerable, y así mismo, establecer el esquema normativo de orden nacional e internacional.

Empero, se ha observado que el estudio de análisis normativo sería más enriquecedor con la recolección de datos a través de trabajo de campo con operadores de justicia de relación directa en la población vulnerables, a través del cual se busca promover mecanismos más eficaces en la exigencia de los derechos fundamentales.

\section{2.- FUNDAMENTO TEÓRICO}

\section{Contexto legal de los grupos vulnerables.}

En el desarrollo de este tema, primero se debe entender el concepto de vulnerabilidad. De primera instancia partiremos del concepto vulnerable, deriva del latín vulnerabilis denota la palabra vulnus que significa herida y abilis que expresa posibilidad, es decir, el adjetivo de situación de riesgo, daño o peligro, sensible e indefenso (Ruiz Rivera, 2011). Cabe referir la vulnerabilidad humana y la vulnerabilidad típica, en ésta última se clasifican la vulnerabilidad social, vulnerabilidad económica, vulnerabilidad atípica o jurídica (Uribe Arzate \& González Chávez, 2007).

En primera instancia cabe establecer que la vulnerabilidad se da en el contexto o circunstancia de mayor indefensión de una situación que presenta una persona, un grupo o una comunidad ya sea por factores sociales, culturales, económicos y físicos (Pérez Contreras, 2005). Algunos autores (Beltráo, y otros, 2014) (Uribe Arzate \& González Chávez, 2007) refieren a los grupos vulnerables a los migrantes, las comunidades en situación de pobreza, comunidades indígenas y comunidades lésbico, gay, bisexual, transexual, transgénico, travesti o intersexual (LGBTTT). De manera general la vulnerabilidad se encuentra vinculada a la discriminación y la desigualdad. 
Bajo este concepto las personas vulnerables se caracterizan por ser incapaces y frágiles, de ahí se considera a los niños, niñas y adolescentes, las mujeres, personas adultas y comunidades indígenas o pueblos indígenas, éstos últimos denominados por el Instituto Interamericano de Derechos Humanos (2018), quienes tienen una cosmovisión nativa propia conforme a sus culturas ancestrales, describe que tienen la protección del derecho como "grupos sujeto de derecho colectivo". Ahora bien, es necesario considerar que atendiendo los momentos o circunstancias de un determinado evento cualquier persona puede constituirse como vulnerable.

Siguiendo este orden de ideas, la vulnerabilidad se entiende en la situación social que resulta de un evento característico en una persona o grupo (Ruiz Rivera, 2011). De tal manera los enfoques teóricos metodológicos reconocen la presencia de dos elementos esenciales: el primero refiere la intensidad de peligro y el segundo la relación con la situación en la cual se encuentra (Cutter, 1996). Por tal motivo es necesario determinar quienes integran los grupos en situación de vulnerabilidad ya que la doctrina discrepa en las connotaciones establecidas en los contextos legales.

Bajo este concepto, algunos autores reconocen la vulnerabilidad primaria establecida para alguna limitación física en la condición humana y la vulnerabilidad secundaria referente a la situación de riesgo que deriva de la condición social y económica (Uribe Arzate \& González Chávez, 2007).

En el marco legal, la reforma del 2011 al artículo $1^{\circ}$. Constitucional ha sido un parteaguas en la vida jurídica, cuya finalidad lata sensu es combatir la violencia, la discriminación y desigualdad en la población mexicana fortaleciendo el compromiso en la evolución social y los derechos humanos. El mencionado artículo sustenta en el párrafo quinto lo siguiente:

"Queda prohibida toda discriminación motivada por origen étnico o nacional, el género, la edad, las discapacidades, la condición social, las condiciones de salud, la religión, las opiniones, las preferencias sexuales, el estado civil o cualquier otra que atente contra la dignidad humana y tenga por objeto anular o menoscabar los derechos y libertades de las personas".

La Carta Magna es muy amplia en este precepto, interpretar la Ley es de manera sui géneris (García Máynez, 2009), en cuanto debemos interpretar toda expresión que establezca un sentido, tales como la expresión, la significación, la conceptualización así como el objeto que persigue. En el referido precepto entendemos como grupos vulnerables a todos aquellos que por sus condiciones religiosas, económicas, sociales y culturales son vulnerados en sus derechos 
humanos.

Por lo tanto, el común denominador en los sujetos en situación de vulnerabilidad es la "desigualdad" de hecho y de derecho que trastoca el acceso a la justicia en nuestra sociedad, porque no se establece la procedencia en la aplicación desde la Constitución garante de la igualdad a los grupos vulnerables.

En este contexto, cabe realizar un estudio profundizado a la integración de los Derechos Humanos de jure para robustecer la temática de estudio es necesario plantear los derechos fundamentales como garantías procesales (Ghys, 2019), materiales o sustantivas, en miras de garantizar la seguridad procesal de los vulnerables.

\section{Contexto Internacional}

Podemos mencionar que los principales Tratados Internacionales en materia de derechos humanos son la Declaración Universal de los Derechos Humanos (1948), la Convención Americana sobre Derechos Humanos en Materia de Derechos Económicos, Sociales y Culturales (Naciones Unidas, 1998) y el Pacto Internacional de Derechos Civiles y Políticos.

Dentro del estatuto que establecen las Naciones Unidas en el tema de estudio del presente trabajo cabe mencionar los siguientes: Declaración Universal de los Derechos Humanos (1948), Pacto Internacional de los Derechos Civiles y Políticos (1966), Convención sobre la eliminación de todas las formas de Discriminación contra la mujer (1979), Convención sobre los Derechos del Niño (1989), Declaración sobre los Derechos de las personas pertenecientes a minorías nacionales o étnicas, religiosas y lingüísticas (1992), Declaración sobre la eliminación de la violencia contra la mujer (1993), Convención Belem Do Pará (1995), Protocolo Adicional a la Convención Americana sobre Derechos Humanos en Materia de Derechos Económicos, Sociales y Culturales “Protocolo de San Salvador" (1998), Declaración de las Naciones Unidas sobre los Pueblos indígenas (2006), Convención sobre los derechos de las personas con Discapacidad (2008), Convención Interamericana para la protección de los Derechos Humanos de las personas mayores (2015).

En consonancia, el artículo 3 del Protocolo adicional a la Convención Americana sobre Derechos Humanos en materia de derechos Económicos, Sociales y Culturales "Protocolo de San Salvador" se sustenta la obligación que tiene el Estado de salvaguardar la no discriminación y garantizar el ejercicio de derechos de las personas. Siguiendo este orden de ideas, el Pacto Internacional de Derechos Civiles y Políticos (1966) reconoce a los derechos como iguales e inalienables, e inherentes a toda la familia humana (Naciones Unidas, s.f.). 
Ahora bien, la Convención sobre la eliminación de todas las formas de Discriminación contra la mujer establecida en 1979, así como la Declaración sobre la eliminación de la violencia contra la mujer (Naciones Unidas, 1993), se reconoce como único instrumento internacional vinculante y reafirma el principio a la no discriminación, de tal manera que advierte que la comisión de discriminación de cualquier acto contra la mujer pone en evidencia la violación de los principios de igualdad y respeto a los derechos y de respeto a la dignidad humana (MirandaMedina, y otros, 2019).

Sin dejar de mencionar que en el año de 1995 el Organismo Internacional (Naciones Unidas), estableció la Convención Belem do Pará con el objetivo de erradicar la violencia de género, empero, para el Estado Mexicano ha representado un gran desafío ante este fenómeno cultural y persistente. Según datos del Instituto Nacional de Geografía se señala que el $6.1 \%$ de las mujeres mayores de 15 años han sufrido algún tipo de agresión (INEGI, 2016) (INEGI, EneroAbril 2017). Así mismo, de jure los informes de los Estados ponen en evidencia la inexistencia de la igualdad.

En las últimas décadas se ha pronunciado la falta de mecanismos y de prevención, así como la carencia en la planeación de programas e infraestructura en los tres ámbitos de gobierno local, estatal y federal (Martínez Pérez \& Sauceda Villeda, 2018) (Miranda-Medina, 2019).

Siguiendo en este orden de ideas, la Declaración sobre los Derechos de las personas pertenecientes a minorías nacionales o étnicas, religiosas y lingüísticas reconoce como derechos fundamentales la dignidad y la igualdad de derechos entre hombres y mujeres (Naciones Unidas, 1992).

De manera colateral la Declaración de las Naciones Unidas sobre los Pueblos indígenas reafirma los Derechos humanos reconocidos en el Derecho Internacional hacia los pueblos indígenas; el artículo 7.1 recalca lo siguiente: "Las personas indígenas tienen derecho a la vida, la integridad fisica y mental, la libertad y la seguridad de la persona" (Naciones Unidas, 2007).

Por su parte, la Convención sobre los Derechos del Niño ratificada por el Estado Mexicano (Naciones Unidas, 1989), es reconocida universalmente como el primer tratado Internacional de obligatoriedad que reconoce los derechos humanos de todos los niños, niñas y adolescentes, conceptualizando la descripción de niño refiriendo que es todo menor de 18 años. La consideración primordial se contempla en el artículo tercero al manifestar el interés superior de la niñez, de tal forma, permite afirmar una supraprotección dejando al Estado la obligación de garantizar el respeto a los derechos igualitarios en un marco de seguridad jurídica. 
Para Miguel Cillero en el estudio del interés superior del menor se deben considerar diversos aspectos antes de tomar cualquier decisión respecto a ellos en una función garantista se deben adoptar aquellas que protejan sus derechos, entre las cuales el referido autor menciona la colaboración en la interpretación de la norma jurídica y la importancia de la creación de políticas públicas que incentiven los derechos de los niños (Cillero Bruñol, 1998).

Por su parte, la Convención sobre los derechos de las personas con Discapacidad establecida por las Naciones Unidas en el 2008, deja de manifiesto la obligación que adquiere el Estado de velar y proteger los derechos humanos de las personas con discapacidad en ella se establecen diversos aspectos iuris et de iure, entre los cuales se encuentran la no discriminación, la igualdad de oportunidades, la accesibilidad, la igualdad entre hombres y mujeres, la inclusión a la sociedad y el respeto (Naciones Unidas, 2008). Algunos autores (Lechuga Cardozo, 2018), suman la seguridad pública y atención médica.

En este contexto, en promoción de los Derechos fundamentales de las personas mayores las Naciones Unidas establece para los Estados partes la Convención Interamericana para la protección de los Derechos Humanos de las personas mayores (1978) (2015), donde se observan diversos aspectos a seguir con este grupo vulnerable como la igualdad, no discriminación, la autorrealización, seguridad física económica y social con la finalidad de erradicar cualquier acto de discriminación así como contribuir en la total inclusión, participación e integración en las comunidades. Cabe advertir que el Estado Mexicano no ha ratificado dicho documento, dejando en evidencia su preocupación el pasado mes de junio del 2018 en el marco de actividades del Día Mundial de Toma de Conciencia de Abuso y Maltrato en la Vejez.

Esta Convención establece diversos conceptos entre los cuales destacan el abandono, discriminación múltiple, discriminación por edad en la vejez, maltrato, negligencia y persona mayor, contempla también el derecho a una vida libre de violencia. De tal forma denota la necesidad de enmarcarlos en normas adjetivas que integran los grupos sociales para contribuir en la certeza jurídica.

Empero, el Estado Mexicano ha contribuido en la creación de normas jurídicas de jurisdicción local (H. Congreso de la Unión , 2002), se evidencia la necesidad de instrumentalizar una Ley adjetiva de ejercicio garante a los derechos fundamentales de las personas débiles y grupos vulnerables en contribución de los deberes substantivum lege. 
Marco jurídico Nacional.

A partir de la reforma Constitucional del 10 de junio del 2011 se observa un nuevo paradigma de justicia al sustentar los derechos humanos en la Norma Suprema de la Nación y con ello la creación de nuevas leyes de ámbito local y Nacional que protegen los derechos fundamentales de los vulnerables. Bajo este contexto, cabe mencionar la Ley de los derechos de las personas adultas mayores (2002), Ley de Seguridad Nacional (2005), Ley General de acceso de las mujeres a una vida libre de violencia (2007), Ley General para la inclusión de las personas con discapacidad (2011), Ley General de los Derechos de Niños, Niñas y Adolescentes (2014), el Estatuto Orgánico del Instituto Nacional de las Personas Adultas Mayores (2014), Ley General de Víctimas (2017), Ley del Instituto Nacional de los Pueblos Indígenas (2018), entre algunas por mencionar.

Con el objeto de velar por la protección de los derechos humanos y el respeto a las garantías individuales, se expide la Ley de Seguridad Nacional (H. Congreso de la Unión, 2005). Y en atención a los Protocolos Internacionales establecidos por la Organización de la Naciones Unidas donde el Estado Mexicano forma parte, objetos de estudio en el apartado anterior, se expiden la Ley General de acceso de las mujeres a una vida libre de violencia (H. Congreso de la Unión, 2007), donde se establecen los principios y modalidades para garantizar el desarrollo a una vida libre de violencia.

Ahora bien, en protección de los menores de 18 años la Ley General de los Derechos de Niños, Niñas y Adolescentes (H. Congreso de la Unión, 2014), contempla los principios del interés superior del menor, universalidad, interdependencia, indivisibilidad y progresividad, igualdad sustantiva, principio pro persona, autonomía progresiva, la no discriminación, la inclusión, además de garantizar la protección y respeto a los derechos humanos. La normativa jurídica en el artículo 4 describe la discriminación múltiple en los menores de la siguiente manera:

"Discriminación Múltiple: La situación de vulnerabilidad especifica en la que se encuentran niñas, niños y adolescentes que, al ser discriminados por tener simultáneamente diversas condiciones, ven anulados o menoscabados sus derechos" ...

Ahora bien, la discriminación múltiple también es conocida como discriminación interseccional (Martínez Fernando, 2008), de tal forma se entiende como aquella que sufre una persona con varios motivos discriminatorios. Sin embargo, algunos autores (Schieck \& Lawson, 2011), establecen diferencia entre los conceptos "discriminación múltiple", "doble discriminación", "triple discriminación", "discriminación compuesta", "discriminación interseccional”. Cabe 
mencionar que la Asamblea General de las Naciones Unidas a través de la Convención de los Derechos de las personas con discapacidad, establece que las mujeres y niñas con discapacidad son más vulnerables al estar sujetas a "múltiples formas de discriminación".

Cabe advertir que, en el Estado Mexicano, no se encuentra contemplada de manera expresa por la Carta Magna la discriminación múltiple, sin embargo, se interpreta dentro artículo $1^{\circ}$. Constitucional. En este sentido, es necesario realizar un análisis profundo de cada uno de los conceptos y contribuir en el fortalecimiento de la creación de nuevas normas jurídicas.

Ahora bien, La Comisión Nacional de Derechos Humanos (CNDH) contempla como grupos vulnerables a las mujeres, niñas, niños y adolescentes, personas en situación de pobreza, personas con discapacidad comunidades indígenas y comunidades lésbico, gay, bisexual, transexual, transgénero, travesti o intersexual (LGBTTT) (Lara Espinosa, 2015). Ante este contexto, la Ley General de Víctimas numeral 5 párrafo décimo reconoce la necesidad de dar una atención especializada a los grupos vulnerables y lo establece de la siguiente manera (H. Congreso de la Unión, 2017):

"Enfoque diferencial y especializado. - Esta Ley reconoce la existencia de grupos de población con características particulares o con mayor situación de vulnerabilidad en razón de su edad, género, preferencia u orientación sexual, etnia, condición de discapacidad y otros, en consecuencia, se reconoce que ciertos daños requieren de una atención especializada que responda a las particularidades y grado de vulnerabilidad de las víctimas".

De las reflexiones descritas en este apartado podemos entender que la situación vulnerable tiene relación con la situación de desventaja, y que incluso podemos encontrar una doble vulnerabilidad cuando en un grupo colectivo integra a su vez a una persona en situación de desventaja. Nuevamente se evidencia la necesidad de establecer un instrumento jurídico de carácter descriptivo y coadyuvar en el corpus iuris de los vulnerables, en función de atender a los grupos débiles y proteger sus derechos humanos.

\section{Los derechos procesales en los grupos vulnerables.}

Los Derechos fundamentales se encuentran fundamentados en los Tratados Internacionales, así como en la Constitución Mexicana, donde se determina la protección de igualdad en distintos sectores minoritarios de las comunidades, para tal efecto el principio de igualdad se entiende en dos vertientes, la primera incurre a la igualdad procesal de jure y la siguiente 
refiere a la no discriminación.

Ahora bien, la terminología observa grandes desafíos ya que, aunque se considera la existencia de suficientes mecanismos jurídicos de especial protección para los grupos débiles y vulnerables imperan actos de discriminación en la vida diaria (Comisión Nacional de Derechos Humanos, 2018). Se deja en evidencia que no se encuentran protegidas en sus derechos. Dentro de este contexto, se debe establecer el concepto de Derechos Humanos. Al respecto, el Instituto Interamericano de los Derechos Humanos (IIDH), establece la siguiente concepción:

"Exigencias elementales que puede plantear cualquier ser humano por el hecho de serlo y que tienen que ser satisfechas porque se refieren a unas necesidades básicas, cuya satisfacción es indispensable para que puedan desarrollarse como seres humanos. Son unos derechos tan básicos que sin ellos resulta difícil llevar una vida digna. Son universales, prioritarios e innegociables".

Los derechos humanos protegen circunstancias, sentidos, momentos que se consideran fundamentales para las personas. Para algunos autores, las principales características es que los derechos fundamentales son absolutos, universales e inalienables (Álvarez Gálvez, 2014).

Es importante remarcar que los derechos humanos de observancia en los procesos jurídicos que atiende grupos vulnerables requieren una vía procesal tuitiva urgente. Así los reconoce el Instituto Interamericano de Derechos Humanos: "A los Estados les corresponde garantizar todos los derechos de sus habitantes por medio de un sistema de garantías judiciales conocido como justicia constitucional". Siguiendo este orden de ideas, el Estado Mexicano establece el control de Convencionalidad y el control de Constitucionalidad en función de salvaguardar el régimen jurídico que acontece los derechos fundamentales de los grupos débiles y personas en vulnerabilidad.

Ahora bien, la Declaración Universal de los Derechos Humanos señala que los derechos humanos son universales, inalienables, irrenunciables, intransferibles, imprescriptibles, e indivisibles. Así mismo, el multicitado artículo $1^{\circ}$. de la Constitución asume la obligación de respetar los derechos a través de los principios de Universalidad, Interdependencia, Indivisibilidad y Progresividad que a continuación se describen.

\section{Principio de Universalidad.}

Este principio sustenta la aplicación de los derechos humanos urbi et orbi a todas las personas por igual sin ninguna distinción ni discriminación, así lo establece el párrafo quinto del artícu- 
lo $1^{\circ}$. Constitucional:

"Queda prohibida toda discriminación motivada por origen étnico o nacional, el género, la edad, las discapacidades, la condición social, las condiciones de salud, la religión, las opiniones, las preferencias sexuales, el estado civil o cualquier otra que atente contra la dignidad humana y tenga por objeto anular o menoscabar los derechos y libertades de las personas".

Se traduce a que todas las personas sin importar su edad, raza, preferencia sexual o nacionalidad son titulares de los derechos fundamentales, no obstante, la experiencia empírica establece que para lograr la igualdad deben considerarse las situaciones que atañen a las personas. Desde este punto de vista, González Amuchastegui (2004), considera dos vertientes, integra la primera como paradigma moral de validez universal y la segunda reconoce la condición de sujeto universal.

\section{Principio de Interdependencia e Indivisibilidad.}

El principio de interdependencia también es conocido como indivisibilidad, es decir que el goce de un derecho vincula a los demás derechos fundamentales, civiles, económicos, sociales y culturales, sin jerarquía entre ellos. De tal manera, ninguno de los derechos es más importantes que otros, tampoco debe considerarse como elementos aislados, contrario sensu deben considerase como un conjunto parte esencial de un todo (Comisión Nacional de Derechos Humanos, 2018).

Siguiendo este orden de ideas, el principio de indivisibilidad manifiesta que ningún derecho puede prescindirse de ningún otro derecho, esto es, no pueden ser jerarquizados o divididos, así en las poblaciones vulnerables se consolidan el respeto a las garantías, la protección de los derechos fundamentales y la exigibilidad de ellos al Estado para su aplicación.

\section{Principio de Progresividad.}

El principio de progresividad solicita que la actuación del Estado proceda en el cumplimiento de los derechos de una manera expedita y eficaz, con la finalidad de satisfacer los derechos económicos, sociales, culturales, civiles y políticos. Deben cumplirse de manera gradual, con las herramientas posibles, necesarias y oportunas, de manera constante y continua en miras de evitar obstaculizar el proceso de las personas vulnerables. 
Cabe mencionar que es responsabilidad de toda la ciudadanía contribuir en el desarrollo de un proceso judicial en protección a los derechos humanos.

Teorías constitucionales de los derechos fundamentales.

El autor García Máynez, refiere el Derecho como el conjunto de normas jurídicas coercitivas de carácter atributivo que en determinado país o época se declaran obligatorios (García Máynez, 2009). Recalcamos el ius poenale y ius piniendi característico en el Derecho positivo.

Por su parte, el Derecho Constitucional "se refiere a la Constitución su valor y eficacia"; entonces, el Constituyente se encarga de estructurar la mejor ingeniería que se puede establecer para su organización procesal y generar por parte del Estado las normas especiales. Por ello compete al Constituyente establecer en forma correcta las beses generales para que no ocurra el desorden, confusión, caos o desgobierno, en la parte que corresponda a crear un aparto de justica completo (Prieto Sanchís, 2018).

Por lo que efectivamente, la inclusión de un Derecho Constitucional significa no sólo la creación normativa realizada por la Ley o Norma Superior creada por el legislador, sino que es necesario que tome como base valores morales.

Así, el Derecho procesal nace de las exigencias sociales, entendido éste como un conjunto de reglas que establecen los requisitos y los efectos de aquél, y esa regulación se da por el lado de la forma, o derecho formal, siendo tales normas sub especie de la norma jurídica, constando así tal derecho de normas y mandatos concretos, autónomos o complementarios, el cual debiera llamarse derecho instrumental (Carnelutti, 2007), determina que a juicio del legislador serán imperativas.

De tal manera, las exigencias sociales y mundiales establecen nuevas formas de resolver los conflictos sociales, donde específicamente en temas de sujetos débiles y vulnerables se somete al escrutinio del impulso social por grupos que exigen igualdad ante la ley para obtener esa justicia en libertad de obstáculos procesales, porque no sólo se trata de un derecho general, sino de un derecho "instrumental" en el que se establezcan mecanismos que coadyuven en las circunstancias específicas de los sujetos procesales.

En México el debido proceso y los derechos humanos como se ha reconocido internacionalmente se fusionan a través de las garantías individuales, de esta manera, la igualdad, la libertad, la audiencia, la defensa, el juicio justo, y la protección de los derechos se elevan al rango derechos fundamentales. Refiere el carácter universal la protección a tales derechos, con una aplicación vinculante internacionalmente a que se adopte la protección más amplia en pro de 
las personas bajo el criterio pro homine que se establece en el referido artículo $1^{\circ}$. de la Constitución de los Estados Unidos Mexicanos.

A fin de completar un marco idóneo de derecho de los sujetos débiles y vulnerables en el ámbito procesal en el Estado Mexicano, se considera necesario el conocimiento y aplicación en la ingería estructural de las normas procesales, nos referimos a la Teoría Iusnaturalista, la Teoría del Bloque Constitucional o de derecho humanos del autor Héctor Fix Zamudio, del derecho natural y su moral que no se puede desligar en su teoría Iusnaturalista o de Derechos Humanos del autor Norberto Bobbio, la Teoría del Garantismo de Luigi Ferrajoli, involucrada con la Tutela Judicial. Con ello se busca hacer efectivo los derechos procesales ante la desigualdad en el valor que la población vulnerable exige.

\section{La teoría Iusnaturalista.}

En primer orden cabe mencionar que "la teoría iusnaturalista" del autor Norberto Bobbio, afirma literalmente "la superioridad del derecho natural sobre el derecho positivo”, (Bobbio, 2015), siendo sostenida ésta superioridad que distinguen tres formas típicas de iusnaturalismo; el escolástico, el racionalista moderno y el Hobbesiano; siendo de aplicación desde la interpretación para el legislador, desde el derecho natural que dictamina respecto del racionalismo moderno como dictamina rectae razón.

La teoría de Hobbes o criterio Hobbesiano que aduce el derecho natural queda reducido a una única norma, por ende el derecho natural es el fundamento o sostén de todo el orden jurídico positivo; que se refiere a que entonces el derecho natural debe obedecer a principios éticos, que debe tomar el legislador como "sujeto humano", el cual debe emitir sus reglas procesales que se positiven como derecho; éste último según la conocida exposición de Santo Tomás, procede de lo que es natural por conclusionem o determinationem” (Bobbio, 2015).

En este contexto, retomamos la teoría de Bobbio en presente artículo dado que es de interés al presente estudio, ya que se pondera el derecho natural, razón previa al establecimiento de normas y tutela de cierta forma los derechos y valores naturales, acertando de una manera clara ante el derecho igualitario y humano, aunque parezca que supera la norma positiva, dando así un juicio real de valor acorde a las condiciones humanas de las personas.

Por ello, la Teoría iusnaturalista robustece el tema de estudio al considerar procesos humanitarios y otorgar juicios valorativos en la escala de valores hacia la población vulnerable (Vigo, 2016). 


\section{La teoría del Bloque Constitucional.}

La teoría del Bloque Constitucional referida por los autores Héctor Fix Zamudio y Salvador Valencia Carmona, en su libro "Las Reformas en Derechos Humanos, Procesos Colectivos y Amparo como nuevo paradigma constitucional" conocida así en el Derecho Supra Nacional, como "cláusula de interpretación de los derechos humanos", se asume una teoría ad hoc a los derecho humanos, en virtud de garantizar los derechos procesales de los grupos vulnerables en virtud de que los criterios de interpretación son a la luz del Derecho comparado o supra nacionales (Fix-Zamudio \& Valencia Carmona, 2013).

Siguiendo este orden de ideas, el Bloque de Constitucionalidad detalla la aplicación obligatoria de aplicar las normas, principios o reglas, Tratados de Derechos Humanos, y otros Tratados Internacionales, los cuales no se encuentran consagrados en la Constitución Política, mismos que deben que ser tomados en consideración al aplicarse en algún proceso o la tutela judicial o jurisdiccional, con el objetivo de que sea efectiva.

De tal manera, se busca fortalecer el pleno ejercicio de los derechos de los adultos mayores, niños, niñas y adolescentes, mujeres y pueblos indígenas desde otros distintos ámbitos sociales, mediante programas políticos y sociales que fomenten su desarrollo pleno, la igualdad, la no discriminación, la independencia, así como la independencia.

El reconocimiento de la existencia de un bloque de constitucionalidad implica identificar todas las normas integrando principios, reglas y valores que materialmente son constitucionales.

\section{La teoría Garantista.}

La Teoría del Garantismo del autor Luigi Ferrajoli, en su libro Principia Iuris, Teoría del Derecho, en cuyas páginas aborda el tema del "garantismo" sustenta la protección que realizan las normas secundarias a la luz de la protección de los derechos humanos. Refiere la garantía primaria señalando la obligación de prestación y obligación del derecho a la acción abstracta en el derecho de petición y la obligación en su caso de prohibición de algún acto, para referirse también a la garantía secundaria como la anulación o condena dispuestas en la garantía de anulabilidad (Ferrajoli, 2011).

Ahora bien, se consideran garantías primarias aquellos mecanismos de salvaguarda que establece el Estado a través de los órganos competentes en protección de la vida, la libertad y los derechos. Se reconoce como garantías secundarias aquellas que protegen los derechos (Ferrajoli, 2011), es decir, la exigencia de parte del Estado a constituir garantías en protección los intereses y necesidades de los sujetos débiles. Bajo este contexto, cabe referir la obligación del Estado en 
la creación de normas instrumentales que tutelen los derechos fundamentales mediante el establecimiento jurídico de las garantías primarias y secundarias a favor de los grupos vulnerables.

\section{La teoría de tutela judicial efectiva.}

El derecho a la tutela judicial efectiva comprende el derecho que tienen las partes de participar en el proceso a través de los órganos jurisdiccionales (Marinoni, 2007) (Salinas Garza , 2016), de ahí la obligación del Estado en materialización efectiva de los derechos fundamentales.

De tal forma, es importante que se tutelen efectivamente los derechos, ante la herramienta de los medios de defensa, del debido proceso y de la sentencia razonada, ello es que las partes débiles o vulnerables puedan defender en forma igualitarias sus derechos, dando con ello inclusión de un modelo procesal publicista otorgando a los juzgadores un papel más activo, y el juez asuma la tutela ante el proceso adjetivo (Salinas Garza , 2016).

Dicha Teoría compagina con la mencionada teoría del garantismo de Ferrajoli, donde el Estado debe tutelar los derechos e intereses sociales y asumir en el Derecho procesal la necesidad de incluir en la Constitución Política de los Estados Unidos Mexicanos a los Grupos Débiles y Vulnerables y posteriormente asentarlos en las leyes instrumentales.

\section{3.- MÉTODO}

\section{Diseño}

El proyecto de estudio se desarrolló con un enfoque cualitativo (Hernández Sampieri, Fernández Collado, \& Baptista Lucio, 2014), de análisis de discurso a través de la heurística (Cárcamo Vásquez, 2005), en la que se interpretan las tres categorías: a) derechos procesales, b) los grupos vulnerables y, c) las normativas nacionales e Internacionales, a través de la reflexión a la Declaración Universal de los Derechos Humanos, la Convención Americana sobre Derechos Humanos en Materia de Derechos Económicos, Sociales y Culturales y el Pacto Internacional de Derechos Civiles y Políticos.

\section{Instrumentos}

En la elaboración del sustento teórico y legal que integra los derechos procesales, los grupos vulnerables y las normativas nacionales e internacionales, se consultaron diversos textos bibliográficos, se incluyen los tratados Internacionales que fundamentan los derechos esenciales de los sujetos vulnerables. También se analizaron Leyes y Protocolos de índole nacional y universal, así como el Seminario Judicial de la Federación del estado mexicano. Se utiliza un análisis crítico valorativo de carácter argumentativo, cuya finalidad es indagar la norma jurídica (Álvarez Undurraga, 2014), 


\section{Procedimiento}

En la determinación del problema de investigación parte desde un análisis de tipo reflexivo y analítico jurídico. Se sustenta a través de la creación de un marco teórico y legal conformado por Tratados Internacionales vinculantes al Estado Mexicano. Bajo este contexto, se realiza una análisis de discurso en el estudio de textos legales en cuanto existen discrepancias en el significado de las normas jurídicas nos encontramos en la tarea de descubrir la intención del legislador (García Máynez, 2009), característica esencial de los secuaces de la exégesis. Posteriormente se analizan los resultados y las conclusiones.

\section{4.- RESULTADOS}

De primer contexto, respecto a los derechos procesales se encontró que la función jurisdiccional se encuentra regulada en la Constitución, sin embargo, se entiende dentro de un esquema subjetivo, ya que impone la necesidad de acudir ante el órgano jurisdiccional para la exigencia de los derechos. Atendiendo el marco jurídico internacional, la Declaración Universal de los Derechos Humanos establece el respeto a los derechos fundamentales a través de los principios de Universalidad, Interdependencia, Indivisibilidad y Progresividad, en este sentido, los derechos humanos se utilizan para dirimir cualquier tema que involucra la protección a la dignidad humana, esto es a los grupos débiles y personas vulnerables.

En cuanto a los grupos vulnerables encontramos que la norma nacional carece de una definición clara y precisa de esta población, ya que el artículo $1^{\circ}$. Constitucional únicamente refiere el concepto de "igualdad" vinculándose al término de "discriminación"; delimitando a "origen étnico o nacional, el género, la edad, las discapacidades, la condición social, las condiciones de salud, la religión, las opiniones, las preferencias sexuales y el estado civil”.

Así mismo, el Pacto Internacional de Derechos Civiles y Políticos asume una diversidad de derechos individuales entre ellos refiere el derecho de igualdad entre hombre y mujeres y el derecho de igualdad ante la Ley. Sin embargo, partiendo de las cualidades especiales de los sujetos que participan en los procesos judiciales se debe igualar al corpus iuris por sus condiciones específicas, estableciendo en contexto de sujetos procesales con igualdad de armas, apoyados en una sistemática jurídica ad hoc a las condiciones de los sujetos de estudio.

Por su parte, la Convención Americana sobre Derechos Humanos en Materia de Derechos Económicos, Sociales y Culturales "Protocolo de San Salvador", reconoce la obligación de los Estados de no discriminar, pero no conceptúa definición de vulnerable o grupos vulnerables. Así mismo, se encontró que existen discrepancias en los diversos conceptos de discriminación, tales como "discriminación múltiple”, “doble discriminación”, "triple discriminación”, “dis- 
criminación compuesta”, "discriminación interseccional”. Por lo tanto, no se debe delimitar únicamente la noción de discriminación, entonces le corresponde al Estado establecer las condiciones necesarias en el goce y disfrute de una vida digna y plena en los derechos humanos.

\section{5.- CONCLUSIONES}

En cuanto a los derechos procesales el precepto constitucional referido en el artículo $1^{\circ}$. requiere fortalecer la descripción legal, ya que no reconoce de manera formal una relación de derechos fundamentales en las minorías lato sensu lo deja a la interpretación, entendiendo la integración en grupos minoritarios tales como grupos étnicos, religiosos, culturales, los inmigrantes, refugiados, tribus y pueblos indígenas.

En la cúspide de los derechos procesales establecidos por las Naciones Unidas se distingue el common law en los grupos vulnerables. Sin embargo, es importante delimitar las nociones de vulnerabilidad directa y vulnerabilidad indirecta para efectos de fortalecer el marco Constitucional, en virtud de homologar la definición de sujetos vulnerables y grupos vulnerables.

En nuestro vértice más inminente se reconoció como valores de los derechos fundamentales la dignidad, libertad e igualdad. Se observó la existencia de un proceso en el cual a todos los sujetos de derecho en las Leyes adjetivas se conceden la misma normativa jurídica de defensa en el debido proceso, ya que "el núcleo duro" lleva implícito ciertas garantías que aplican a cualquier procedimiento de naturaleza jurisdiccional y son las que la Suprema Corte de Justicia de la Nación ha identificado como formalidades esenciales del procedimiento. Empero, es necesario continuar con la creación de nuevas técnicas procesales para efectos de coadyuvar con el derecho procesal en los grupos vulnerables.

Se concluye que existe la necesidad de generar un protocolo para la atención de derechos procesales en los grupos vulnerables en protección de los derechos humanos. Así mismo, se propone generar nuevos estudios de jurisdicción constitucional a favor de los sujetos débiles y en vulnerabilidad.

\section{REFERENCIAS}

Álvarez Gálvez, Í. (2014). Universales, absolutos e inalienables: los derechos indestructibles. Revista de humanidades de Valparaíso(14), 63-80. 
Álvarez Undurraga, G. (2014). La investigación jurídica como un instrumento de mejoramiento del proceso de enseñanza-aprendizaje del Derecho. Revista Pedagogía Universitaria y Didáctica del Derecho, I(1), 36-57.

Beltráo, F. J., Monteiro de Brito, J., Gómez, I., Pajares, E., Paredes, F., \& Zúñiga, Y. (2014). Derechos Humanos de los grupos Vulnerables. Manual. Barcelona: Universitat. Popmpeu Fabra. Comisión Europea.

Bobbio, N. (2015). Iusnaturalismo y Positivismo Jurídico. Madrid, España: Editorial Trotta.

Cárcamo Vásquez, H. (2005). Hermenéutica y Análisis Cualitativo. Revista de Epistemología de Ciencias Sociales, 204-216.

Carnelutti, F. (2007). Teoría General del Derecho. España: Ediciones Olejnik.

Cillero Bruñol, M.(1998). El interés superior del niño en lemarco de la Convención Internacional sobre los Derechos del Niño. Obtenido de www.iin.oea.org/IIN/cad/Participacion/pdf/el_ interes_superior.pdf

Colombo Campbell, J. (2002). Funciones del Derecho Procesal Constitucional. Ius et Praxis, $8(2), 1-30$.

Comisión Nacional de Derechos Humanos. (2018). Análisis cuantitativo y cualitativo de las Violaciones de los Derechos Humanos de Grupos Vulnerables. Comisión Nacional de los Derechos Humanos. Obtenido de www.cndh.org.mx

Comisión Nacional de Derechos Humanos. (2018). Los Principios de Universalidad, Interdependencia, Indivisibilidad y progresividad de los Derechos Humanos. Obtenido de www.cndh.org.mx/sites/all/doc/cartillas/2015-2016/34-Principios-universalidad.pdf

Cutter, S. L. (1996). Vulnerability toenvironmental hazards. Progress in Human Geography, 20(4), 529-539.

Ferrajoli, L. (2011). Principia Iuris, Teoría del Derecho. Bologna Italia: Editorial Trotta.

Fix-Zamudio, H., \& Valencia Carmona, S. (2013). Las Reformas en Derechos Humanos, Porcesos Colectivos y Amparo como nuevo Paradigma Constitucional. México: Porrúa.

García Máynez, E. (2009). Introducción al Estudio del Derecho (61 ed.). México: Porrúa.

Ghys, T. (2019). Abuso espectacular y sistemático de derechos humanos en México. Revista Política, Globalidad y Ciudadanía, 5(9), 17-24. 
González Amuchastegui, J. (2004). Autonomía, dignidad y ciudadanía. Valencia: Tirant lo Blanch.

H. Congreso de la Unión . (2002). Ley de los Derechos de las personas adultas mayores. Obtenido de www.diputados.gob.mx/LeyesBiblio/pdf/245_120718.pdf

H. Congreso de la Unión. (2005). Ley de Seguridad Nacional. Obtenido de www.diputados. gob.mx/LeyesBiblio/pdf/LSegNac.pdf

H. Congreso de la Unión. (2007). Ley General de Acceso de las Mujeres a una vida libre de violencia. Obtenido de www.diputados.gob.mx/LeyesBiblio/pdf/LGAMVLV_130418.pdf

H. Congreso de la Unión. (2014). Ley General de los Derechos de Niñas, Niños y adolescentes. Obtenido de www.diputados.gob.mx/LeyesBiblio/pdf/LGDNNA_200618.pdf

H. Congreso de la Unión. (03 de Enero de 2017). Ley General de Víctimas. Obtenido de www. diputados.gob.mx/LeyesBiblio/pdf/LGV_030117.pdf

H. Congreso de la Unión. (s.f.). Constitución Política de los Estados Unidos Mexicanos. Obtenido de www.diputados.gob.mx

Hernández Sampieri, R., Fernández Collado, C., \& Baptista Lucio, P. (2014). Metodología de la Investigación. México: McGraw-Hill.

INEGI. (2016). Encuesta Nacional de Victimizacion y Percepcion de la Seguridad Pública. Obtenido de http://www.inegi.org.mx/saladeprensa/boletines/2016/especiales/ especiales2016_09_04.pdf

INEGI. (Enero-Abril 2017). Fuentes y datos de la violencia contra las mujeres en México. Aprendizajes, dificultades y retos acumulados. Realidad Datos y espacio. Revista Internacional de Estadística y Geografía, 8(1), 6-16.

Lara Espinosa, D. (2015). Grupos en situación de vulnerabilidad. México: Comisión Nacional de los Derechos Humanos.

Lechuga Cardozo, J. I. (2018). Necesidades insatisfechas a una comunidad aledaña a una terminal portuaria de Barranquilla 1. Pensamiento y Gestión(45), 181-210.

Marinoni, L. G. (2007). Derecho Fundamental a la tutela jurisdiccional efectiva. Lima, Perú: Palestra Editores SAC.

Martínez Fernando, R. (2008). La discriminación múltiple, una realidad antigua, un concepto nuevo. Revista Española de Derecho Constitucional, $28,84$. 
Martínez Pérez, Y. B., \& Sauceda Villeda, B. J. (2018). Los MASC desde el sistema para el Desarrollo Integral de la Familia en México. Revista de la Facultad de Derecho(44), 1-31. doi:http://dx.doi.org/10.22187/rfd2018n44a12

Miranda-Medina, C. F. (2019). Estudio bibliométrico en justicia restaurativa. En O. P. Lugo Serrato, C. Cedalice Riquelme, G. Gorjón Gómez, \& R. Soler Mendizabal, La Transversalidad de los MASC. Una perspectiva México Panamá (págs. 421-441). Monterrey: Editorial Universitaria UANL; Corte Suprema de Justicia de Panamá.

Miranda-Medina, C. F., Martínez Pérez, Y. B., García Salvador, K., Hernández Martínez, A., Rodríguez Medrano, K., \& Sosa Ruíz, M. (2019). Percepción sobre la violencia en las relaciones amorosas de una Facultad de Derecho y Criminología en una Universidad del Norte de México.Erg@omnes Revista jurídica, 11(1),171-195.

Naciones Unidas. (10 de Diciembre de 1948). La Declaración Universal de los Derechos Humanos. Obtenido de www.un.org/es/documents/udhr/

Naciones Unidas. (11 de Febrero de 1978). Convencion Americana sobre los Derechos Humanos. Obtenido de Organizacion de las Naciones Unidas. Pacto de San José: https:// www.oas.org/dil/.../1969_Convención_Americana_sobre_Derechos_Humanos...

Naciones Unidas. (20 de Noviembre de 1989). Convención sobre los Derechos del Niño. Obtenido de https://www.unicef.org/mexico/spanish/mx_Convencion_Derechos_es_final. pdf

Naciones Unidas. (18 de Diciembre de 1992). Declaración sobre los derechos de las personas pertenecientes a minorías nacionales o étnicas, religiosas y lingüisticas. Obtenido de https://www.ohchr.org/SP/ProfessionalInterest/Pages/Minorities.aspx

Naciones Unidas. (1993). Declaración sobre la eliminación de violencia contra la mujer. Obtenido de https://www.ohchr.org/sp/professionalinterest/pages/violenceagainstwomen. aspx

Naciones Unidas. (1995). Convención Belem Do Pará. Obtenido de https://www.oas.org/dil/ esp/convencion_belem_do_para.pdf

Naciones Unidas. (1998). Protocolo Adicional a la Convención Americana sobre Derechos Humanos en materia de Derechos Económicos, Sociales y Culturales "Protocolo de San Salvador". Obtenido de www.ordenjuridico.gob.mx/TratInt/Derechos\%20Humanos/PI2. pdf

Revista Política, Globalidad y Ciudadanía, Vol. 6. No. 12, Julio - Diciembre 2020, Universidad Autónoma de Nuevo León, Monterrey, México, ISSN 2395-8448. 225-245. http://revpoliticas.uanl.mx/index.php/RPGyC/article/view/126 
Naciones Unidas. (13 de Septiembre de 2007). Declaración sobre los Derechos de los Pueblos indígenas. Obtenido de https://www.un.org/esa/socdev/unpfii/documents/DRIPS_es.pdf

Naciones Unidas. (2008). Convención sobre los Derechos de las personas con Discapacidad. Obtenido de https://www.un.org/esa/socdev/enable/documents/tccconvs.pdf

Naciones Unidas. (2015). Convención Interamericana sobre la protección de los derechos humanos de las personas mayores. Obtenido de www.oas.org > ... > Tratados y Acuerdos » > Tratados Multilaterales Interamericanos »

Naciones Unidas. (s.f.). Pacto Internacional de Derechos Civiles y Políticos. Obtenido de www.ohchr.org > OHCHR > Español > Interés profesional

Peláez Grisales, H. (2015). Una mirada al problema del derecho de los sujetos y grupos desventajados de especial protección en Colombia y la apuesta por una necesaria fundamentación teórica desde las teorías contemporáneas de la justicia. Revista de Estudios Socio-Jurídicos, 17(1), 125-168.

Pérez Contreras, M. M. (2005). Aproximación a un estudio sobre vulnerabilidad y violencia familiar. Boletín mexicano de derecho comparado(113), 845-867.

Prieto Sanchís, L. (2018). Constitucionalismo y Positivismo. Lima, Perú: Palestra, Editores.

Ruiz Rivera, N. (2011). La definición y medición de la vulnerabilidad social. Un enfoque normativo. Investigaciones Geográficas, boletín del Instituto de Geografia(77), 63-74.

Salinas Garza, J. Á. (2016). Tutela Judicial Efectiva. Una visión Constitucional y Convencional de la Teoría del Proceso. Ediitorial Liber Iuris Novum.

Schieck, D., \& Lawson, A. (2011). European Union Non-Ddiscrimination Law and Intersectionality. Investigating the Triangle of Racial, Genaer and Disability Discrimination. New York: Farnham: Ashgate.

Uribe Arzate, E., \& González Chávez, M. L. (2007). La protección jurídica de las personas vulnerables. Revista de Derecho(27), 205-229.

Vigo, R. L. (2016). Constitucionalización y Judicialización del Derecho. México, D.F.: Porrúa. 